\title{
An integrated stroke system in Hong Kong
}

Hong Kong Med J 2018;24:318

DOI: 10.12809/hkmj187413

To the Editor-There have been repeated calls to establish a coordinated acute stroke system in Hong Kong. ${ }^{1,2}$ Overseas experience demonstrates that intravenous thrombolysis (IVT) can be reliably administered at a mean of 52 minutes from alerting prehospital services. ${ }^{3}$ This study from Berlin ${ }^{3}$ utilising ambulances equipped with computed tomography scanners (among other system changes in care delivery) may have little resemblance to the current situation in Hong Kong. Nonetheless it shows what is possible with appropriate vision, determination, and resources.

Similar to other time critical interventions, the speed and coverage of IVT in the stroke system must be balanced with the potential risks and harm for our patients. An integrated system will lead to stroke mimics (SM)-patients who present with nonvascular neurological conditions that closely resemble stroke-presenting in greater numbers to the emergency department. These patients are often ignored in the planning of acute stroke services as they have far more impact on emergency physicians than stroke physicians. Therefore, the impact of establishing an acute stroke system on emergency departments must be factored into the planning of these services at the outset.

We concur with Leung ${ }^{1}$ and the findings of a recent meta-analysis that systems change interventions can increase the proportion of eligible stroke patients receiving IVT. ${ }^{4}$ An integrated approach between prehospital providers, emergency department staff, and stroke physicians will improve outcomes. The establishment of stroke centres with clear protocols for primary diversion will require coordinated multidisciplinary input, and with careful consideration given to the resources required for the triage, transport, investigation, and ongoing care of SM patients who will inevitably be diverted to stroke centres inadvertently.

\section{Declaration}

All authors have no conflicts of interest to disclose.

Kevin KC Hung, FHKCEM, FHKAM (Emergency Medicine)

Colin A Graham *, MD, FHKCEM

Accident and Emergency Medicine Academic Unit, The Chinese University of Hong Kong, Shatin, Hong Kong

* Corresponding author: cagraham@cuhk.edu.hk

\section{References}

1. Leung GK. We need a stroke system. Hong Kong Med J 2018;24:9-10.

2. Graham CA. Rational emergency stroke care in Hong Kong. Hong Kong Med J 2012;18:262-3.

3. Ebinger $M$, Winter $B$, Wendt $M$, et al. Effect of the use of ambulance-based thrombolysis on time to thrombolysis in acute ischemic stroke: a randomized clinical trial. JAMA 2014;311:1622-31

4. Paul CL, Ryan A, Rose S, et al. How can we improve stroke thrombolysis rates? A review of health system factors and approaches associated with thrombolysis administration rates in acute stroke care. Implementation Sci 2016;11:51. 